\title{
Inhaltsverzeichnis
}

\author{
(Anschließend das Gesetzesregister)
}

\section{Die Fälle}

(S. $1-36$ des Heftes in der Schlaufe des hinteren Deckels)

\section{Die Lösungen zum Ersten Teil der Fälle}

\author{
(S. $39-315)$ \\ Systematische Vorbemerkungen

A. Die Tatbestandsmäßigkeit . . . . . . . . . . . . . 39-40

I. Ihre Bestandteile. . . . . . . . . . . . 39-40

I. Die Handlung . . . . . . . . . . . . . . 39

2. Die objektiven Tatbestandsmerkmale . . . . 39-40

3. Das subjektive Unrechtselement. . . . . . . . 40

4. Der Kausalzusammenhang . . . . . . . . . $4^{\circ}$

II. Die Folgen mangelnder Tatbestandsmäßigkeit ............... . 40

B. Die Rechtswidrigkeit . . . . . . . . . . . . . . $4 \mathrm{I}-43$

I. Die Regel................... 4 I

II. Die objektive Rechtswidrigkeit ist kein Tatbestandsmerkmal . . . . . . . . . . $44^{I}$

III. Rechtswidrigkeitsausschließungsgründe . . $4^{\mathrm{I}-43}$

IV. Die Folgen mangelnder Rechtswidrigkeit . 43

C. Die Schuld . . . . . . . . . . . . . . . . 44-58

I. Das Wesen der Schuld ......... . 43

II. Diebiologischen VoraussetzungenderSchuldbejahung . . . . . . . . . . . . . . . I. Die altersmäBige Verstandes- und Willensreife .

2. Die Zurechnungsfähigkeit . . . . . . .

III. Die Schuldformen . . . . . . . . . . . 45-46

I. Der direkte oder unbedingte Vorsatz . . . . 45

2. Der bedingte Vorsatz (dolus eventualis) . . . . 46

3. Der dolus generalis . . . . . . . . . . . . 46

4. Die Fahrlässigkeit. . . . . . . . . . . 46

IV. Die Schuldausschließungsgründe . . . . 47-48

V. Die Folgen eines Schuldausschließungs grundes................. 
VI. Tatbestands - u. Verbotsirrtum . . . . . . 49-57

I. Der Tatbestandsirrtum. . . . . . . . . . . 49-52

2. Der Verbotsirrtum . . . . . . . . . . 52-57

VII. Besondere, für die Schuldfrage bedeutungslose Irrtumsfälle . . . . . . . . . . .

I. Objektverwechslung . . . . . . . . . . . .

2. Irrtum über Kausalverlauf . . . . . . . . . . $57-5^{8}$

. 57

3. Wahnverbrechen .......... . . $57-58$

D. Die persönlichen strafbefreienden Gründe . . . . . 5 5-59

I. Strafausschließungsgründe . . . . . . . $5^{8}$

II. Strafaufhebungsgründe . . . . . . . . . . 59

III. Die Folgen der persönlichen strafbefreienden Gründe. . . . . . . . . . . . . . 59

E. Die objektiven Strafbarkeitsbedingungen . . . . . 59-6I

I. Begriff und Wesen . . . . . . . . . 59

2. Ubersicht . . . . . . . . . . . 59-60

3. Gemeinsame Regeln. . . . . . . . . . . 60

4. Die Behandlung im Proze $B$. . . . . . . . 60-61

F. Die Prozeßvoraussetzungen und Prozeßhindernisse . . $61-62$

1. Begriff und Wesen ............ $6 \mathbf{r}$

2. Die Prozeßvoraussetzungen. . . . . . . . $6 \mathbf{r}$

3. Die Prozeßhindernisse . . . . . . . . . . . 62

4. Gemeinsame Regeln . . . . . . . . . . . . . 62

5. Die Behandlung im Prozeß . . . . . . . . . 62

1. Fall : Ein unerfreulicher Bräutigam . . . . . . . 3

Lösung . . . . . . . . . . . . . . . . . $63-92$

Allgemeiner Teil : Straflose Nachtat S. 67. Der Strafantrag S. 79 und 88-92. Deliktscharakter der „,besonders schweren Fälle“ $\mathrm{S} . \mathbf{8}_{2}$

Besonderer Teil : Betrug (durch Vorenthalten der Wahrheit S. 63; als straflose Nachtat bei vorausgegangener Unterschlagung S. 67; der Vermögensschaden S. 66; der Kreditbetrug S. 68; durch Übereignung einer unterschlagenen Sache S. 7I; durch Zahlung mit ungedecktem Scheck S. $72 \mathrm{f}$; Kausalität zwischen Irrtum und Vermögensverfügung S. 73; durch abredewidrige Ausfüllung eines Blankoakzepts S. 75; durch Wechselfälschung S. 78; Eingehungsbetrug und Erfüllungsbetrug, insbesondere das Problem des Anstellungsbetrugs S. 81; der Vermögensschaden bei sittenwidrigen Geschäften S. 77); Unterschlagung (durch Ableugnen des Besitzes S. 64; Irrtum 
über die Fremdheit der Sache S. 67; durch Sicherungsübereignung einer fremden Sache $S .70$ ). Rückfallvoraussetzungen beim Betrug S. 81. Persönliche Begünstigung bei Strafantragsdelikten S. 83 .

$\S \S 43,59,246,263$; ferner: Art. 10,17 und 69 des Wechselgesetzes.

Ferner: Betrugstatbestände außerhalb des $\S 263$ (insbesondere Versicherungsbetrug und Automatenmißbrauch) S. $84 \mathrm{ff}$. Unterschlagungstatbestände außerhalb des $\S 246$ (Depotunterschlagung) S. $87 \mathrm{ff}$.

2. Fall: Der Nachschlüsseldieb und sein Gehilfe . .

Lösung . . . . . . . . . . . . . . . . . . . 92-1 28

Allgemeiner Teil: Die Teilnahme (allgemeine Erörterungen zu $\S 50$ Abs. I und 2 mit Beispielen) S. $94 \mathrm{ff}$. Die Beihilfe (allgemeine Erörterungen S. 99-104; der vorliegende Fall S. 104). Versuch, Rücktritt und tätige Reue (allgemeine Erörterungen mit Beispielen S. I $7-122$; der vorliegende Fall, insbesondere Rücktritt des Teilnehmers S. $107 \mathrm{ff}$.).

Ferner: Fortgesetzte Tat und Kollektivdelikte (allgemeine Erörterungen) S. I $23 \mathrm{ff}$.

Besonderer Teil: Rückfalldiebstahl S. 93. Betrug durch Verkauf gestohlener Sachen S. 94. Begünstigung (allgemeine Erörterungen S. Io8ff.; der vorliegende Fall S. I I I ff., insbesondere Selbstbegünstigung S. 113 und Begünstigung als Beihilfe S. I14). Hehlerei S. I $15 \mathrm{ff}$.

$\S \S 43,4^{6}, 47,4^{8}, 49,50,24^{\circ}, 243$ I $3,244,245,257$, $258,259,263,369$ Z. I.

3. Fall: Der falsche Kriminalbeamte. . . . . . . . . 7

Lösung . . . . . . . . . . . . . . . 128-154

Allgemeiner Teil : Anstiftung zur Begünstigung bezüglich der eigenen Tat S. 136.

Besonderer Teil: Amtsanmaßung (allgemeine Erörterungen S. 128-1 29; der vorliegende Fall S. 129). Hausfriedensbruch S. I30. Nötigung S. 130. Urkundenfälschung (öffentliche Urkunde S. 130; Mißbrauch von Ausweispapieren S. 132). Diebstahl eines Sparkassenbuchs (allgerneine Erörterungen zum Problem der Zueignungsabsicht S. 132-135; der vorliegende Fall S. 1 35). Sachliche Begünstigung (Unterschied gegenüber Sachhehlerei S. 137-139; der vorliegende Fall S. I39). 
$\S \S 48,123,240,242,257,258,259,263,267,28 \mathrm{r}$. Ferner: Allgemeine Diebstahlserörterungen (einfacher Diebstahl S. I40-I46; schwerer Diebstahl S. 146-150; Rückfalldiebstahl S. 150-151; Besitz von Diebeswerkzeug S. 15I-153; Spezialfälle des Diebstahls S. I53-154.)

4. Fall: Irrtum kann vor Strafe schützen . . . . .

Lösung . . . . . . . . . . . . . . . . . . . 154-161

Allgemeiner Teil: Tatbestandsirrtum S. I6o; Verbotsirrtum S. 16of. Beihilfe gegenüber einem im Irrtum befindlichen Täter S. 16I.

Besonderer Teil: Vollstreckungsvereitelung (allgemeine Erörterungen S. I 54-156; der vorliegende Fall S. 156). Verstrickungsbruch (allgemeine Erörterungen S. I57-159; der vorliegende Fall S. 159-161).

$\S \S 49,59, \quad$ I $37,288$.

5. Fall : Der gewissenlose Rechtsagent . . . . . . . 9 Lösung . . . . . . . . . . . . . . . . . . . $161-198$

Allgemeiner Teil: Untersagung der Berufsausübung S. 184. Eventualdolus bei Hehlerei S. 192.

Besonderer Teil: Mittelbare Falschbeurkundung S. 16Iff. Meineid (allgemeine Erörterungen zu $\S \S$ I53-I63 S. I62 ff.; der vorliegende Fall S. 166). Prozeßbetrug (allgemeine Erörterungen S. I66; der vorliegende Fall S. 167). Untreue (allgemeine Erörterungen S. I68-1 72 ; der vorliegende Fall S. $172-174$ ). Betrug durch Bewirkung eines Verzichts auf eine nichtige Forderung S. I 74. Urkundenfälschung allgemeine Erörterungen S. 175-183; insbesondere Herstellung einer unechten Urkunde S. I79-18I; der vorliegende Fall S. 183-184). Sachhehlerei (allgemeine Erörterungen S. I84-I9I; der vorliegende Fall S. 191 f.).

$\S \S 153 \mathrm{ff} ., 246,259,263,266,267,27$ I.

Ferner: Untreuetatbestände außerhalb des $\$ 266$,

S. 193. Hehlereitatbestände außerhalb des $\S 259$,

S. 194. Die Wahlfeststellung, S. 194. Fälschung von Gesundheitszeugnissen ( $\$ \S 277-279)$ S. $196 \mathrm{ff}$.

6. Fall: Der Münzfälscher Johannes Steidel . . . . 10 Lösung . . . . . . . . . . . . . . . . I98-222 Allgemeiner Teil: Gesetzeskonkurrenz (allgemeine Erörterungen S. 202-205; der vorliegende Fall S. 205 
bis 206). Tateinheit und Tatmehrheit (allgemeine Erörterungen S. 216-218; der vorliegende Fall S. $210 \mathrm{ff}$.). Mittäterschaft bei zweiaktigen Straftaten (allgemeine Erörterungen S. 215-216; der vorliegende Fall S. 216).

Besonderer Teil : Münzverbrechen und Münzvergehen (allgemeine Erörterungen) S. 198-200. Abschieben von Falschgeld S. 200; im Zusammentreffen mit Betrug S. 202-205. Versuchtes Münzverbrechen S. 206. Strafbare Vorbereitungshandlung S. 206-207. Vollendete Falschmünzerei S. 208; im Zusammentreffen mit Betrug S. 210-2I I. Unterschlagung (an Fundsache S. 205; zum Nachteil eines Mittäters S. 209). Verbreitung von Falschgeld S. 212; im Zusammentreffen mit Betrug S. 214 .

$\S \S 47,73,74,146,147,148,149,150,151,152,246$, 263,360 Nr. 4 und 5 .

Ferner: Fälschung und wiederholte Verwendung von Stempel- und Postwertzeichen ( $\S \S 275,276)$ mit Beispielen S. $218-222$.

7. Fall : Der beleidigte Ratsschreiber . . . . . . . .

Lösung . . . . . . . . . . . . . . . 222-240

Allgemeiner Teil: Notwehr (allgemeine Erörterungen S. 237-240, der vorliegende Fall S. 234-235).

Besonderer Teil: Das Vergehen der Beleidigung (allgemeine Erörterungen) S. 222-232. Der vorliegende Fall: Úble Nachrede S. 232; Wahrnehmung berechtigter Interessen S. 233-234; Kompensation S. 234-236; Publikationsbefugnis S. 236. $\S \S 53,61,65,185-200$.

\section{Fall: Die unehelichen Kinder der Luise Baumeister}

Lösung . . . . . . . . . . . . . $240-256$

Allgemeiner Teil: Mittelbare Täterschaft (allgemeine Erörterungen S. 24I-246; der vorliegende Fall S. 251). Verbotsirrtum S. 248-250.

Besonderer Teil : Personenstandsdelikt (allgemeine Erörterungen S. 240-241; der vorliegende Fall S. 247 bis 251). Mittelbare Falschbeurkundung (allgemeine Erörterungen S. 252-254, der vorliegende Fall S. 254 bis 255).

$\S \S 48,59,169,271,272$. 
9. Fall: Ein folgenschwerer Einfall . . . . . . . $\quad 15$

Lösung . . . . . . . . . . . . 256-289

Allgemeiner Teil: Mittäterschaft (allgemeine Erörterungen S. 269-272; der vorliegende Fall S. 272). Aufforderung zum Verbrechen und deren Annahme (allgemeine Erörterungen S. 273; der vorliegende Fall S. 274). Anstiftung (allgemeine Erörterungen S. 274-278; der vorliegende Fall S. 278-280). Sicherungsmaßregeln, insbesondere Sicherungsverwahrung (allgemeine Erörterungen S. 28I-286; der vorliegende Fall S. 286-287).

Besonderer Teil: Erpressung (allgemeine Erörterungen S. 256-259; der vorliegende Fall S. 259). Räuberische Erpressung S. 260-26r. Besonders schwerer Raub S. 262. Körperverletzung mit tödlichem Ausgang S. 262. Fahrlässige Tötung S. 262 . Unterschlagung (an Gegenständen einer Leiche) S. 263 . Brandstiftung (allgemeine Erörterungen S.264 bis 266 ; der vorliegende Fall S. 266-268). Tötungsdelikt S. 268. Nichtanzeige von Verbrechen S. 280 f. Der Raubmord S. 288. Der Autostraßenraub S. 28gf. $\S \S 20 \mathrm{a}, 42 \mathrm{a}, 42 \mathrm{e}, 43,46,47,48,49 \mathrm{a}, 13^{8}, 139,2$ I I, $212,222,226,246,249,250,25$ I, 253, 255, 303, 305, 306, 310, 310a, 316 a.

10. Fall: Der weibliche Trunkenbold . . . . . . . .

Lösung . . . . . . . . . . . . . . . . 291-315

Allgemeiner Teil: Kausalzusammenhang (allgemeine Erörterungen S. 295-300; der vorliegende Fall S. 300-301). Fahrlässigkeit (allgemeine Erörterungen) S. 301-303. Unterlassungsdelikte (allgemeine Erörterungen) S. 305-308. Eventualdolus S. 310-311.

Besonderer Teil: Giftbeibringung (allgemeine Erörterungen S. 29I; der vorliegende Fall S. 291-292). Körperverletzung S. 292-293. Aussetzung (allgemeine Erörterungen S. 293; der vorliegende Fall S. 293-294). Fahrlässige Tötung S. 303; im Zusammentreffen mit Aussetzung mit Todesfolge S. 303 . Mord und Totschlag (allgemeine Erörterungen S. 308 bis 309; der vorliegende Fall S. 309-3 I I). Unterlassene Hilfeleistung (allgemeine Erörterungen S. 312 bis 313; der vorliegende Fall S. 313-314).

$\S \S$ I 38, 139, $211,212,221,222,223,229,330 \mathrm{c}$.

Ferner: Unfallflucht S. 314-315. 